\title{
Antiangiogenic drugs used with chemotherapy for patients with recurrent ovarian cancer: a meta-analysis
}

This article was published in the following Dove Press journal:

OncoTargets and Therapy

17 February 2017

Number of times this article has been viewed

\author{
SuYi Yi \\ Longjia Zeng \\ Yan Kuang \\ ZhiJuan Cao \\ ChengJun Zheng \\ Yue Zhang \\ Meng Liao \\ Lu Yang
}

Department of Gynaecology and Obstetrics, The First Affiliated Hospital of Guangxi Medical University, Nanning, Guangxi, People's Republic of China
Correspondence: Yan Kuang Department of Gynaecology and Obstetrics, The First Affiliated Hospital of Guangxi Medical University, 6 Shuangyong Road, Nanning, Guangxi 530021, People's Republic of China Tel +86 I3877I66099 Email461161401@qq.com
Objective: The value of antiangiogenic inhibitors for patients with recurrent ovarian cancer has not been completely affirmed. Therefore, we aimed to assess the effectiveness and toxicities of various antiangiogenic drugs for the treatment of recurrent ovarian cancer.

Methods: In this meta-analysis, we searched PubMed, EMBASE, and the Cochrane Central Register of Controlled Trials databases for complete randomized controlled trials. The searches were extended to May 15, 2016. The risk of bias of the included studies was evaluated via a Cochrane systematic evaluation, and the statistical analyses were performed using RevMan 5.2 software. Results: In total, we included 8 randomized controlled trials involving 3,211 patients and divided them into 3 groups, vascular endothelial growth factor receptor inhibitors (VEGFRIs), vascular endothelial growth factor (VEGF) inhibitors (bevacizumab), and angiopoietin inhibitors (trebananib). The progression-free survival improved significantly in all the groups being given antiangiogenic drugs (hazard ratio [HR]: 0.55, 95\% confidence interval [CI]: 0.45-0.67, $I^{2}=0 \%, P<0.00001$ for the VEGFRI group; HR: $0.53,95 \%$ CI: $0.45-0.63, P^{2}=51 \%, P<0.00001$ for the VEGF inhibitor group; HR: $0.67,95 \%$ CI: $0.58-0.77, I^{2}=0 \%, P<0.00001$ for the trebananib group). Overall survival was obviously prolonged in the VEGFRI (HR: $0.76,95 \% \mathrm{CI}$ : $0.59-0.97, P^{2}=0 \%, P=0.03$ ), the VEGF inhibitor (HR: $0.87,95 \%$ CI: $0.77-0.99, P^{2}=0 \%, P=0.03$ ), and trebananib groups (HR: $0.81,95 \%$ CI: $0.67-0.99, P=0 \%, P=0.04$ ). The incidence of grade $3 / 4$ side effects was different among the 3 groups, for example, proteinuria, hypertension, gastrointestinal perforation, and arterial thromboembolism were presented in the VEGF inhibitor group. Increased incidences of fatigue, diarrhea, and hypertension were seen in the VEGFRI group, and the trebananib group had a higher incidence of hypokalemia.

Conclusion: This meta-analysis showed that antiangiogenic drugs improved the progressionfree survival. The VEGFRI, bevacizumab, and trebananib groups showed increased overall survival. Adding antiangiogenic drugs to chemotherapy treatment resulted in a higher incidence of grade $3 / 4$ side effects, but these were manageable.

Keywords: antiangiogenesis, recurrent ovarian cancer, progression-free survival, overall survival, toxicity

\section{Introduction}

Currently, ovarian cancer is the leading cause of cancer-related death in middle-aged and elderly females. ${ }^{1}$ Despite the significantly improved prognosis of advanced ovarian cancer, it will recur in $>50 \%$ of women within $18-24$ months. $^{2}$ The treatment of relapsing ovarian cancer mainly consists of a single or a combination of intravenous chemotherapy. The addition of antiangiogenic drugs in the treatment of relapsed ovarian cancer has not yet been fully defined. ${ }^{3}$ 
According to our search results, 8 randomized controlled trials (RCTs) have been conducted on this topic. ${ }^{4-11}$ To the best of our knowledge, there are 2 pathways for neovascularization, including the vascular endothelial growth factor (VEGF) and angiopoietin pathways. VEGF signaling through VEGF receptors (VEGFRs) activated downstream signal transduction molecules phospholipase C- $\gamma(\mathrm{PLC}-\gamma), \mathrm{PI} 3 \mathrm{~K}$, Akt, Ras, Src, and MAPK and regulated cell proliferation, migration, survival, and vascular permeability. ${ }^{10,12-15}$ Therefore, we divided these RCTs into 3 groups, including a VEGF receptor inhibitor (VEGFRI) group, VEGF inhibitor group, and angiopoietin group.

Several meta-analyses have been conducted on a single antiangiogenic drug or advanced ovarian cancer. However, this meta-analysis aimed to estimate the efficacy and toxicity of various antiangiogenic drugs for the treatment of patients with recurrent ovarian cancer.

\section{Methods}

The PubMed, EMBASE, and Cochrane Central Register of Controlled Trials databases were comprehensively searched from January 2000 to May 2016, without language restrictions. The search was limited to RCTs with or without antiangiogenic therapy for recurrent ovarian cancer. The search terms included "ovarian cancer", "ovarian carcinoma”, "ovarian neoplasm”, "ovarian tumor", "angiogenesis", "angiogenic", and "randomized controlled trial". Abstracts from the annual meetings of the American Society of Clinical Oncology, the European Society of Medical Oncology, and the Society of Gynecologic Oncology from within the past five years were also searched.

\section{Study selection and inclusion criteria}

The inclusion criteria were as follows: 1) the research subjects were patients with recurrent ovarian cancer, including platinum-sensitive and platinum-resistant patients; 2) chemotherapy interventions with or without antiangiogenic drugs; and 3) RCTs. The articles were obtained for an independent assessment of eligibility by 2 of the authors (SY Yi and LJ Zeng). A difference of opinion was resolved via consultation with a third author (Y Kuang), if necessary.

\section{Data extraction and quality assessment}

Two of the authors (SY Yi and LJ Zeng) independently extracted the data on the basis of the following: first author, year of publication, age, pathology, sample size, intervention, and outcome data. As shown in Figure 1, we assessed the quality of the eligible studies according to the Cochrane Collaboration's risk of bias tool in the Cochrane Handbook for Systematic

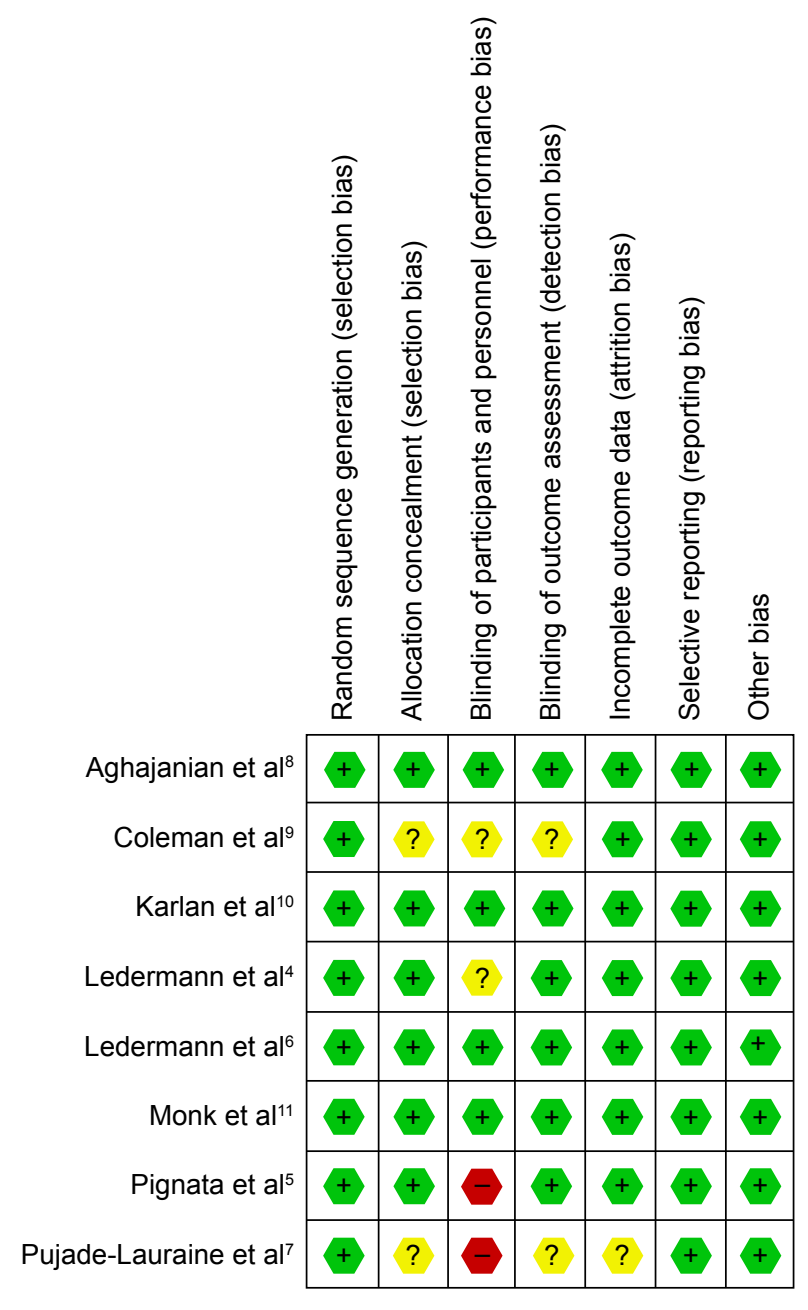

Figure I Assessment of the quality of the included randomized controlled trials: low risk of bias (green hexagons), unclear risk of bias (yellow hexagons), and high risk of bias (red hexagons).

Reviews of Interventions 5.1.0. We resolved any disagreements by discussing them with a third review author (Y Kuang).

\section{Statistical analysis}

The pooled hazard ratios (HRs) and 95\% confidence interval ( $95 \% \mathrm{CI}$ ) for the progression-free survival (PFS) or overall survival (OS) and the relative risks (RRs) for adverse events from all the articles were calculated using RevMan 5.2. The heterogeneity among the studies was estimated using the $I^{2}$ index. We use the fixed-effects model if $I^{2} \leq 50 \%$, otherwise the random-effects model was applied. The statistical analyses were performed using the RevMan 5.2 software.

\section{Results}

\section{Search and study characteristics}

The search process of this study is presented in Figure 2, and the characteristics of the RCTs used are presented in Table 1. After searching the literature, only 8 RCTs containing 3,211 patients 


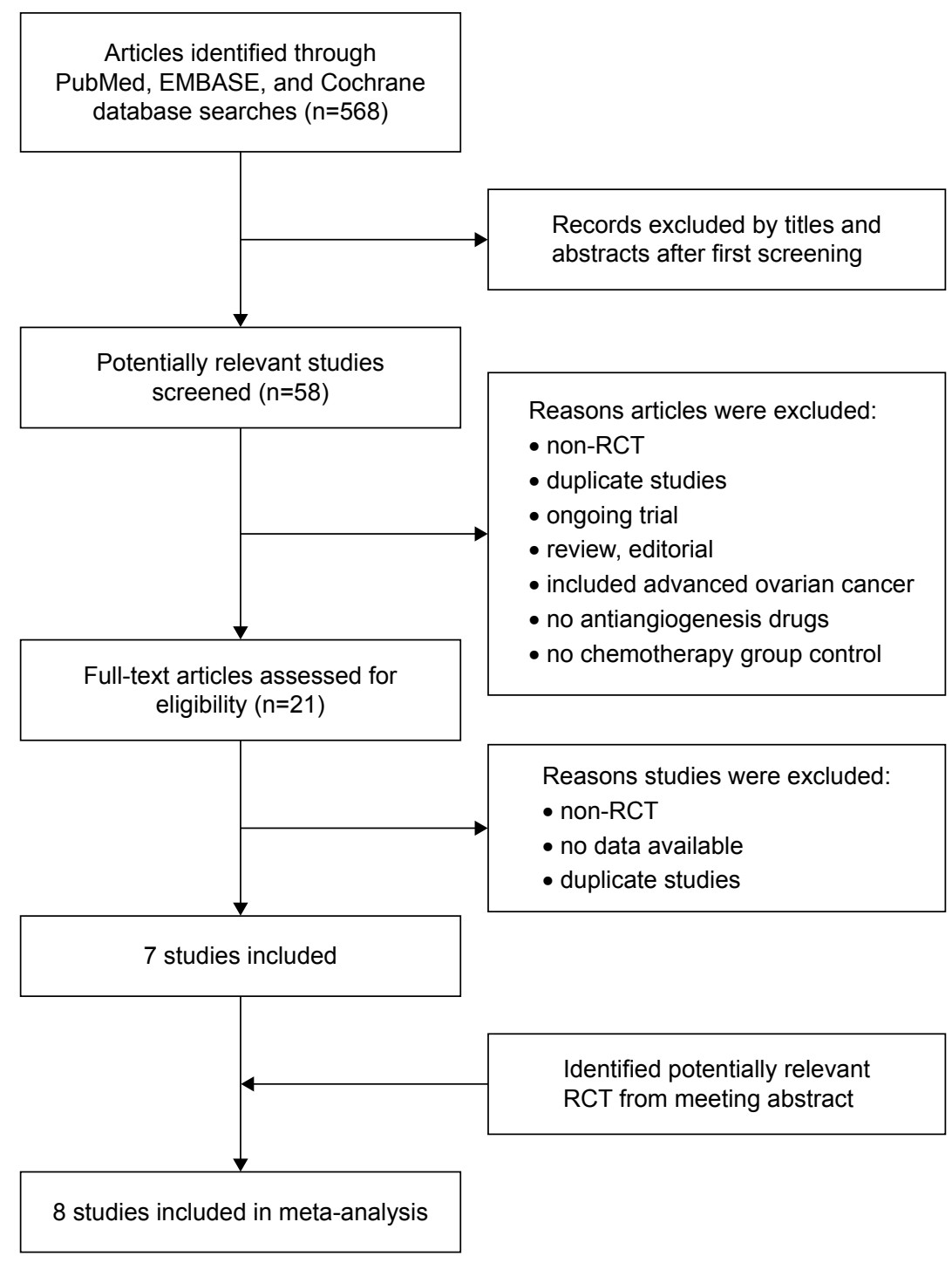

Figure 2 Flowchart showing study selection procedure. Abbreviation: RCT, randomized controlled trial.

with recurrent ovarian cancer were included in this analysis. We divided them into the following 3 groups according to the different active targets of the antiangiogenic drugs: 3 RCTs with VEGFRIs, ${ }^{4-6} 3$ RCTs with VEGF inhibitors, ${ }^{7-9}$ and 2 RCTs with angiopoietin inhibitors. ${ }^{10,11}$ One RCT applied antiangiogenic drugs during the maintenance phase, ${ }^{4}$ but the other drugs were fully employed from the beginning of therapy to disease progression in the other 7 RCTs. The evaluation of the quality of the RCTs included in this study is shown in Figure 1. We were not concerned about publication bias due to the fact that only 8 articles were included. ${ }^{16}$

\section{PFS}

A random-effects model was applied with high heterogeneity, and all the RCTs included reported the HRs and 95\%
CIs of the PFS. As illustrated in Figure 3, compared with chemotherapy alone, the PFS improved significantly in all the groups, as follows: HR: $0.55,95 \%$ CI: $0.45-0.67$, $I^{2}=0 \%, P<0.00001$ for the VEGFRI group; HR: $0.53,95 \%$ CI: $0.45-0.63, I^{2}=51 \%, P<0.00001$ for the VEGF inhibitor group; and HR: $0.67,95 \% \mathrm{CI}$ : $0.58-0.77, I^{2}=0 \%, P<0.00001$ for the trebananib group.

\section{OS}

The OS was determined by using a fixed-effects model attributed to low heterogeneity among the studies. Only 1 study did not report the OS. ${ }^{5}$ The OS was obviously prolonged in the VEGFRI (HR: $0.76,95 \%$ CI: $0.59-0.97, P=0 \%, P=0.03$ ), the VEGF inhibitor group (HR: $0.87,95 \%$ CI: $0.77-0.99$, $I^{2}=0 \%, P=0.03$ ), and the trebananib group (HR: $0.81,95 \%$ 


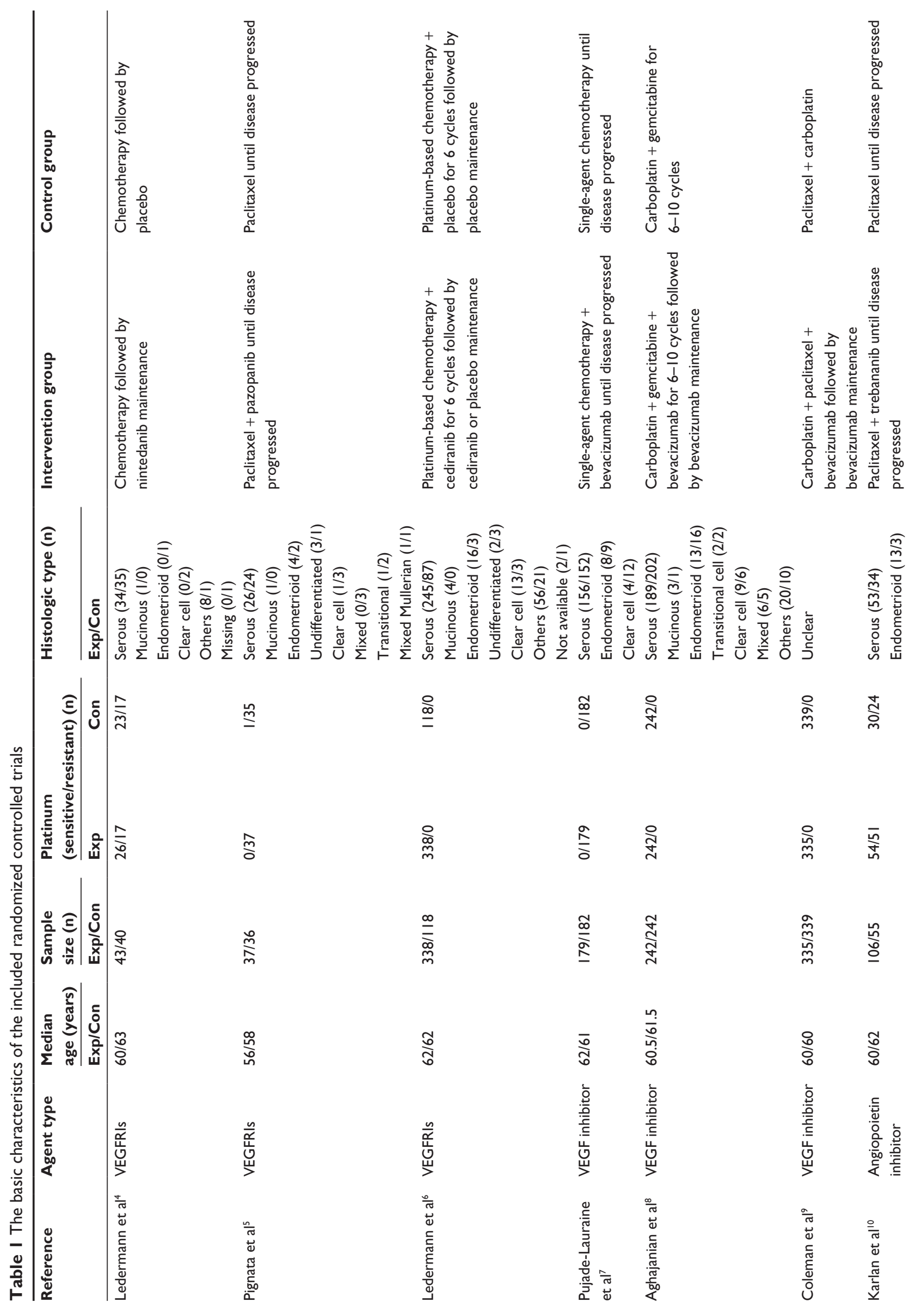




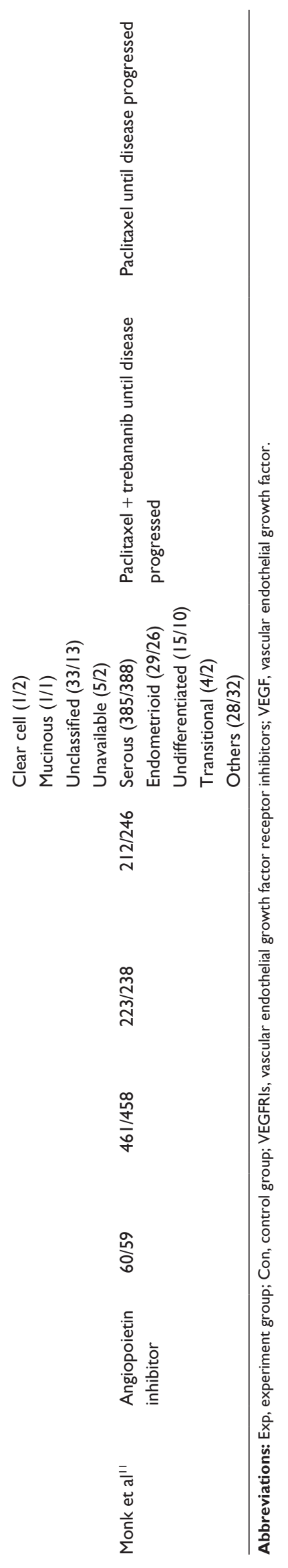

CI: $\left.0.67-0.99, I^{2}=0 \%, P=0.04\right)$. The outcomes are shown in Figure 4.

\section{Toxicity (adverse effect grade $\geq 3$, except gastrointestinal perforation [GIP] grade $\geq \mathrm{I}$ )}

As presented in Figure 5, the rates of hypertension (RR: 3.68, 95\% CI: $1.49-9.07, I^{2}=0 \%, P=0.005$ ), fatigue (RR: $2.08,95 \%$ CI: $1.11-3.87, I^{2}=0 \%, P=0.02$ ), and diarrhea (RR: $5.31,95 \%$ CI: $1.75-16.16, P^{2}=0 \%, P=0.003$ ) were numerically higher in the VEGFRI treatment group. In addition, the nintedanib as a VEGFRI was associated with higher incidences of hepatotoxicity (RR: 20.47, 95\% CI: 2.89-144.88, $P=0.003$ ).

With regard to the VEGF inhibitor group, the proteinuria (RR: $15.64,95 \% \mathrm{CI}$ : $4.87-50.23, I^{2}=0 \%, P<0.00001$ ), hypertension (RR: $12.44,95 \% \mathrm{CI}: 3.62-42.79, I^{2}=32 \%$, $P<0.0001$ ), arterial thromboemboli (RR: $4.84,95 \% \mathrm{CI}$ : 1.24-18.91, $P^{2}=0 \%, P=0.02$ ), and GIP (RR: $3.62,95 \% \mathrm{CI}$ : $\left.2.09-6.26, I^{2}=0 \%, P<0.00001\right)$ were significantly different (Figure 6).

As shown in Figure 7, the trebananib treatment may cause a higher risk of hypokalemia (RR: $2.25,95 \%$ CI: 1.16-4.35, $I^{2}=0 \%, P=0.02$ ).

\section{Subgroup analysis}

We divided the RCTs into 2 subgroups, 2 RCTs with platinum-resistant ${ }^{5,7}$ and 3 RCTs with platinum-sensitive. ${ }^{6,8,9}$ As shown in Figure 8, the PFS improved significantly both the platinum-resistant and the platinum-sensitive recurrent ovarian cancer (HR: $0.47,95 \%$ CI: $0.38-0.58, P^{2}=0 \%$, $P<0.00001$ for the platinum-resistant group; HR: $0.56,95 \%$ CI: $0.50-0.63, P=26 \%, P<0.00001$ for the platinum-sensitive group). As presented in Figure 9, the OS was clearly better in the platinum-sensitive group (HR: $0.86,95 \%$ CI: 0.76-0.99, $P=0.03$ ), with no obvious statistical significance for OS in the platinum-resistant group (HR: $0.81,95 \%$ CI: $0.65-1.02$, $\left.I^{2}=2 \%, P=0.07\right)$.

\section{Discussion}

Ovarian cancer is the leading cause of cancer deaths in women, ${ }^{1}$ and many patients with advanced ovarian cancer will undergo recurrences with chemotherapy resistance. Ovarian carcinoma is a complicated disease, controlled by multiple mutating genes, and there are no clear molecular therapeutic targets. Therefore, a new promising therapy targeting the tumor microenvironment has been proposed. Neovascularization is crucial for tumor growth and 


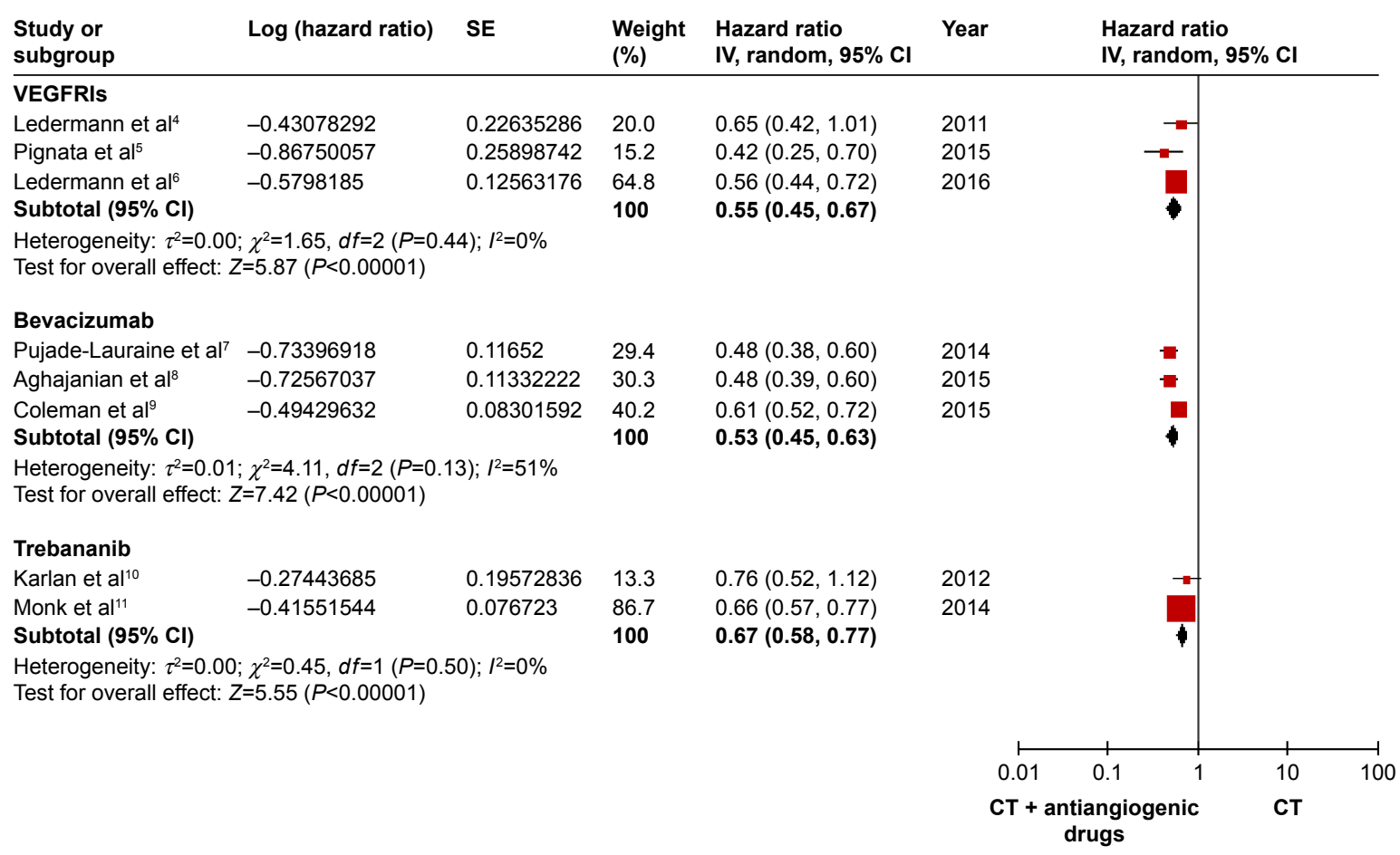

Figure 3 Forest plot of progression-free survival data for different antiangiogenic drugs.

Abbreviations: SE, standard error; CI, confidence interval; IV, instrumental variable; VEGFRIs, vascular endothelial growth factor receptor inhibitors; CT, chemotherapy.

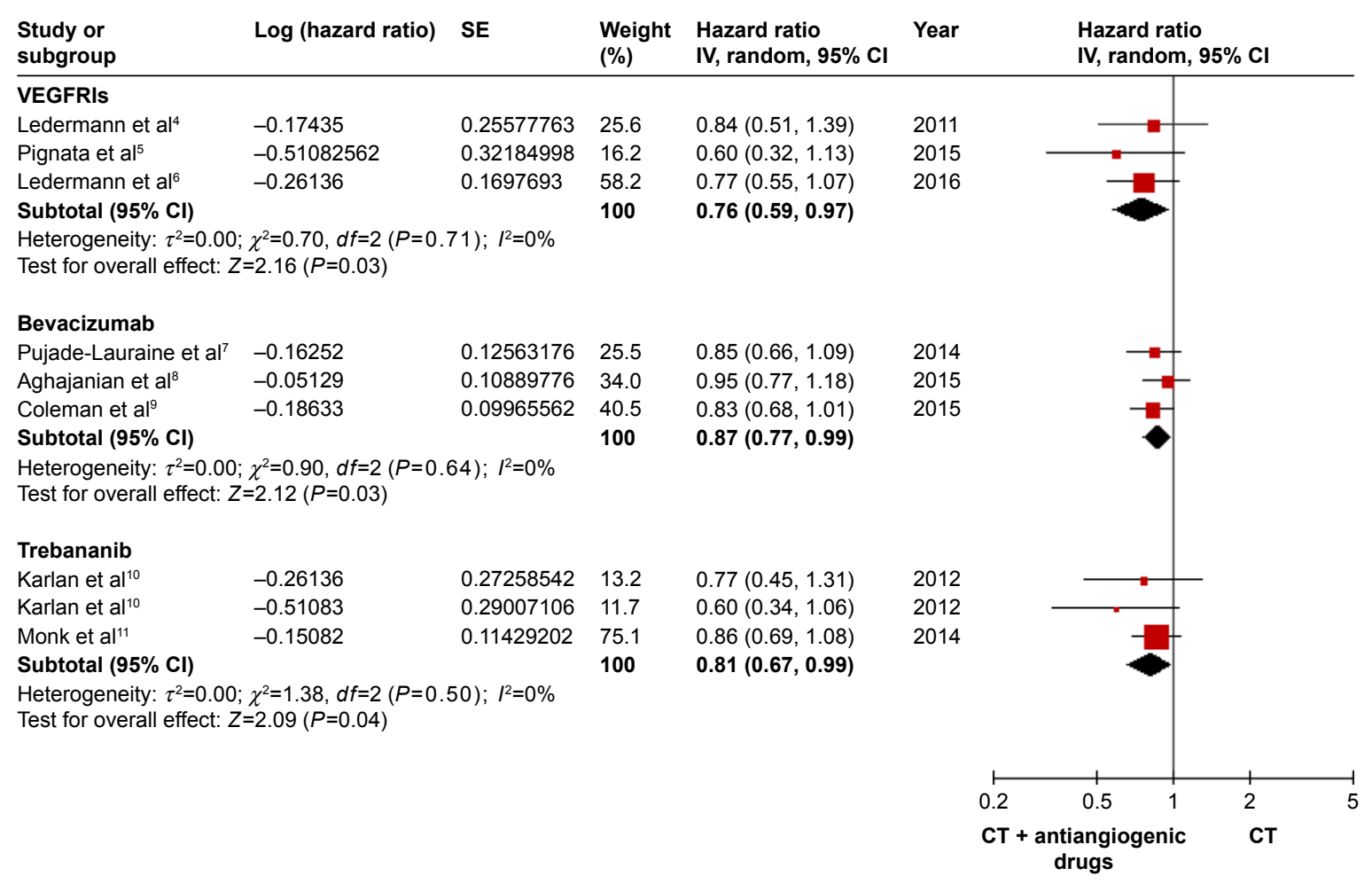

Figure 4 Forest plot of overall survival data for different antiangiogenic drugs.

Abbreviations: SE, standard error; Cl, confidence interval; IV, instrumental variable; VEGFRIs, vascular endothelial growth factor receptor inhibitors; CT, chemotherapy. 


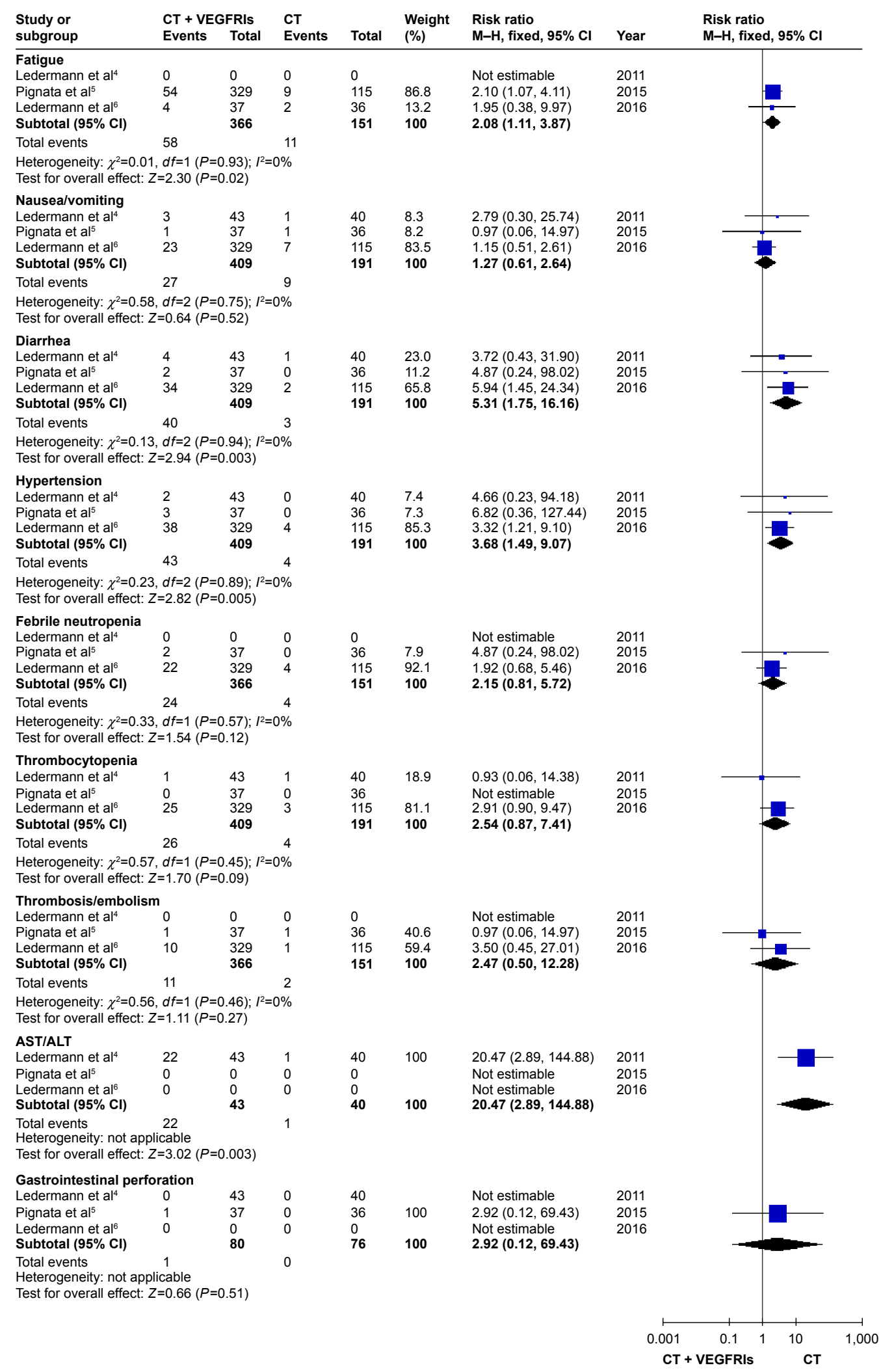

Figure 5 The forest plot for evaluating the toxicity (the VEGFRI group).

Abbreviations: CT, chemotherapy; VEGFRI, vascular endothelial growth factor receptor inhibitor; Cl, confidence interval; AST, aspartate transaminase; ALT, alanine transaminase; $\mathrm{M}-\mathrm{H}$, Mantel-Haenszel. 


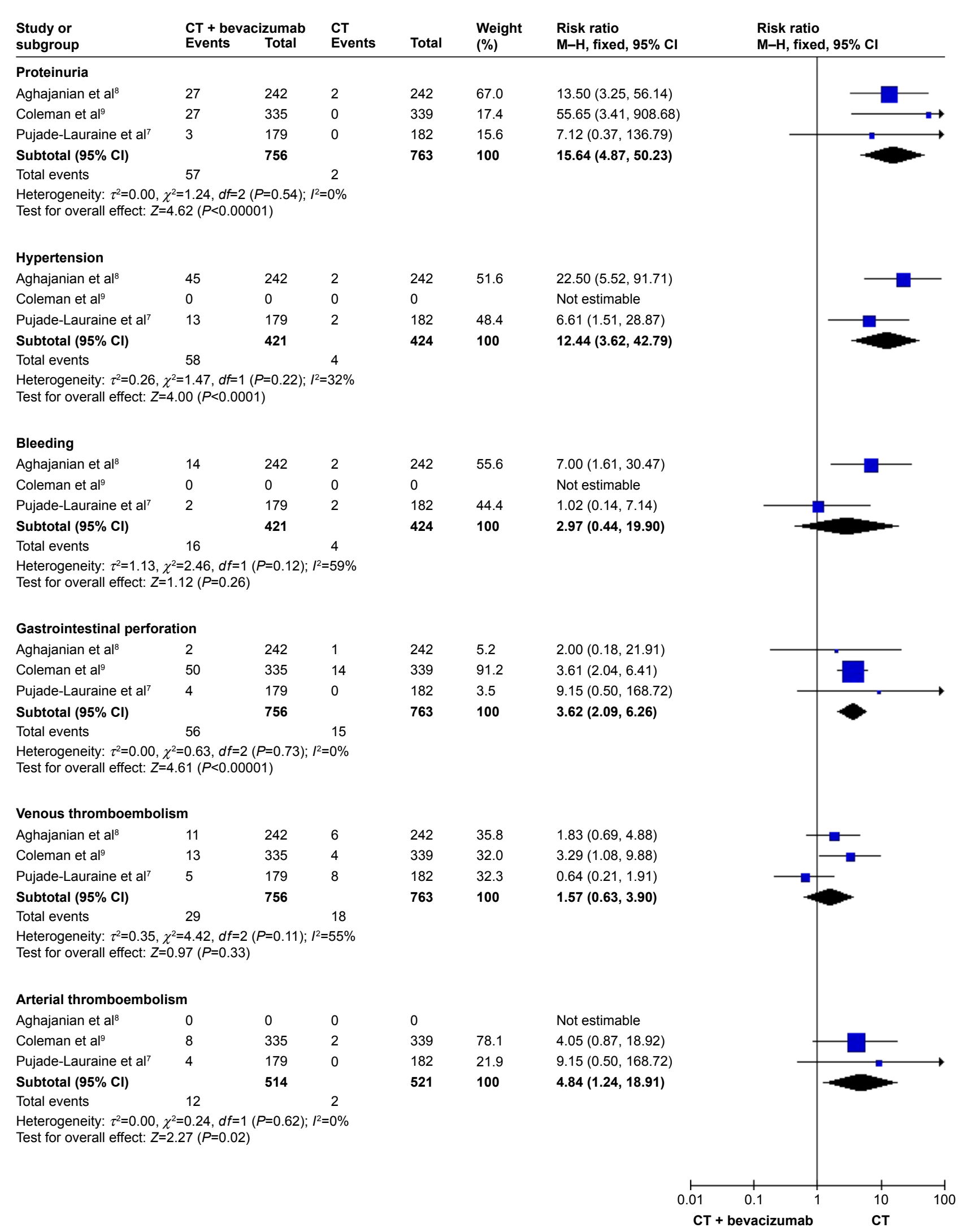

Figure 6 The forest plot for evaluating the toxicity (the bevacizumab group).

Abbreviations: $\mathrm{CT}$, chemotherapy; $\mathrm{Cl}$, confidence interval; $\mathrm{M}-\mathrm{H}$, Mantel-Haenszel. 


\begin{tabular}{|c|c|c|c|c|c|c|}
\hline $\begin{array}{l}\text { Study or } \\
\text { subgroup }\end{array}$ & $\begin{array}{l}\text { CT + treb } \\
\text { Events }\end{array}$ & $\begin{array}{r}\text { nnanib } \\
\text { Total }\end{array}$ & $\begin{array}{l}\text { CT } \\
\text { Events }\end{array}$ & Total & $\begin{array}{l}\text { Weight } \\
\text { (\%) }\end{array}$ & $\begin{array}{l}\text { Risk ratio } \\
\text { M-H, random, }\end{array}$ \\
\hline \multicolumn{7}{|l|}{ Fatigue } \\
\hline Monk et al ${ }^{11}$ & 15 & 461 & 17 & 452 & 85.6 & $0.87(0.44,1.71$ \\
\hline Karlan et al ${ }^{10}$ & 4 & 106 & 2 & 55 & 14.4 & $1.04(0.20,5.49$ \\
\hline Subtotal $(95 \% \mathrm{Cl})$ & & 567 & & 507 & 100 & $0.89(0.47,1.67$ \\
\hline Total events & 19 & & 19 & & & \\
\hline \multicolumn{7}{|c|}{$\begin{array}{l}\text { Heterogeneity: } \tau^{2}=0.00 ; \chi^{2}=0.04, d f=1(P=0.84) ; l^{2}=0 \% \\
\text { Test for overall effect: } Z=0.37(P=0.71)\end{array}$} \\
\hline \multicolumn{7}{|l|}{ Vomiting/nausea } \\
\hline Monk et al ${ }^{11}$ & 22 & 461 & 18 & 452 & 82.3 & $1.20(0.65,2.20$ \\
\hline Karlan et al ${ }^{10}$ & 7 & 106 & 3 & 55 & 17.7 & $1.21(0.33,4.5$ \\
\hline Subtotal $(95 \% \mathrm{Cl})$ & & 567 & & 507 & 100 & $1.20(0.69,2.0$ \\
\hline Total events & 29 & & 21 & & & \\
\hline
\end{tabular}

Heterogeneity: $\tau^{2}=0.00 ; \chi^{2}=0.00, d f=1(P=0.99) ; l^{2}=0 \%$

Test for overall effect: $Z=0.65(P=0.52)$

\section{Edema}

$\begin{array}{lllllll}\text { Karlan et al }{ }^{10} & 5 & 106 & 2 & 55 & 44.9 & 1.30(0.26,6.47)\end{array}$

$\begin{array}{lllll}\text { Subtotal }(95 \% \mathrm{Cl}) & 567 & 507 & 100 & 3.79(0.55,25.92)\end{array}$

Total events $42 \quad 6$

Heterogeneity: $\tau^{2}=1.47 ; \chi^{2}=4.12, d f=1(P=0.04) ; l^{2}=76 \%$

Test for overall effect: $Z=1.36(P=0.17)$

\section{Hypokalemia}

Monk et al ${ }^{11}$

Karlan et a $1^{10}$

21

$21 \quad 461$

461

Subtotal $(95 \% \mathrm{Cl})$

Total events 12

105

$$
2
$$

$\begin{array}{ll}452 & 79.5 \\ 55 & 20.5 \\ 507 & 100\end{array}$

$2.06(0.98,4.32)$

$3.14(0.73,13.55)$

$2.25(1.16,4.35)$

Heterogeneity: $\tau^{2}=0.00, \chi^{2}=0.26, d f=1 \quad(P=0.61) ; l^{2}=0 \%$

Test for overall effect: $Z=2.40(P=0.02)$

Peripheral neuropathy

Karlan et al ${ }^{10} \quad 6$

Monk et al ${ }^{11} \quad 13$

Subtotal $(95 \% \mathrm{Cl})$

Total events

19

105
461
566

$\begin{array}{ll}55 & 23.6 \\ 452 & 76.4 \\ \mathbf{5 0 7} & \mathbf{1 0 0}\end{array}$

$1.57(0.33,7.53)$

$1.59(0.67,3.81)$

$1.59(0.74,3.40)$

Heterogeneity: $\tau^{2}=0.00 ; \chi^{2}=0.00, d f=1 \quad(P=0.99) ; I^{2}=0 \%$

Test for overall effect: $Z=1.19(P=0.23)$

\section{Dyspnea}

Karlan et al ${ }^{10}$

Monk et al11

Subtotal $(95 \% \mathrm{Cl})$

Total events

6
10

105
461
$\mathbf{5 6 6}$

2
6

$\begin{array}{ll}55 & 29.1 \\ \mathbf{4 5 2} & 70.9 \\ \mathbf{5 0 7} & \mathbf{1 0 0}\end{array}$

16

8

Heterogeneity: $\tau^{2}=0.00 ; \chi^{2}=0.00, d f=1(P=0.97) ; l^{2}=0 \%$

Test for overall effect: $Z=1.11 \quad(P=0.27)$

Gastrointestinal perforation

\begin{tabular}{|c|c|c|c|c|c|c|}
\hline Karlan et al ${ }^{10}$ & 0 & 105 & 1 & 55 & 44.4 & $0.18(0.01,4.25)$ \\
\hline Monk et al ${ }^{11}$ & 7 & 461 & 1 & 452 & 55.6 & $6.86(0.85,55.56)$ \\
\hline Subtotal $(95 \% \mathrm{Cl})$ & & 566 & & 507 & 100 & $1.35(0.04,47.93)$ \\
\hline Total events & 7 & & 2 & & & \\
\hline
\end{tabular}

$1.57(0.33,7.53)$

$1.63(0.60,4.46)$

$1.62(0.69,3.76)$

Test for overall effect: $Z=0.16(P=0.87)$

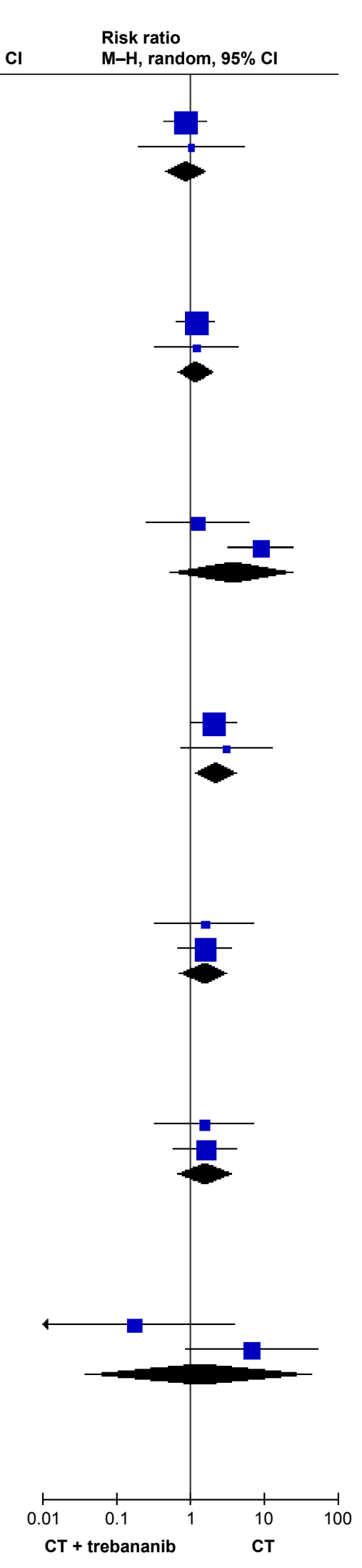

Figure 7 The forest plot for evaluating the toxicity (the trebananib group).

Abbreviations: $\mathrm{CT}$, chemotherapy; $\mathrm{Cl}$, confidence interval; $\mathrm{M}-\mathrm{H}$, Mantel-Haenszel. 


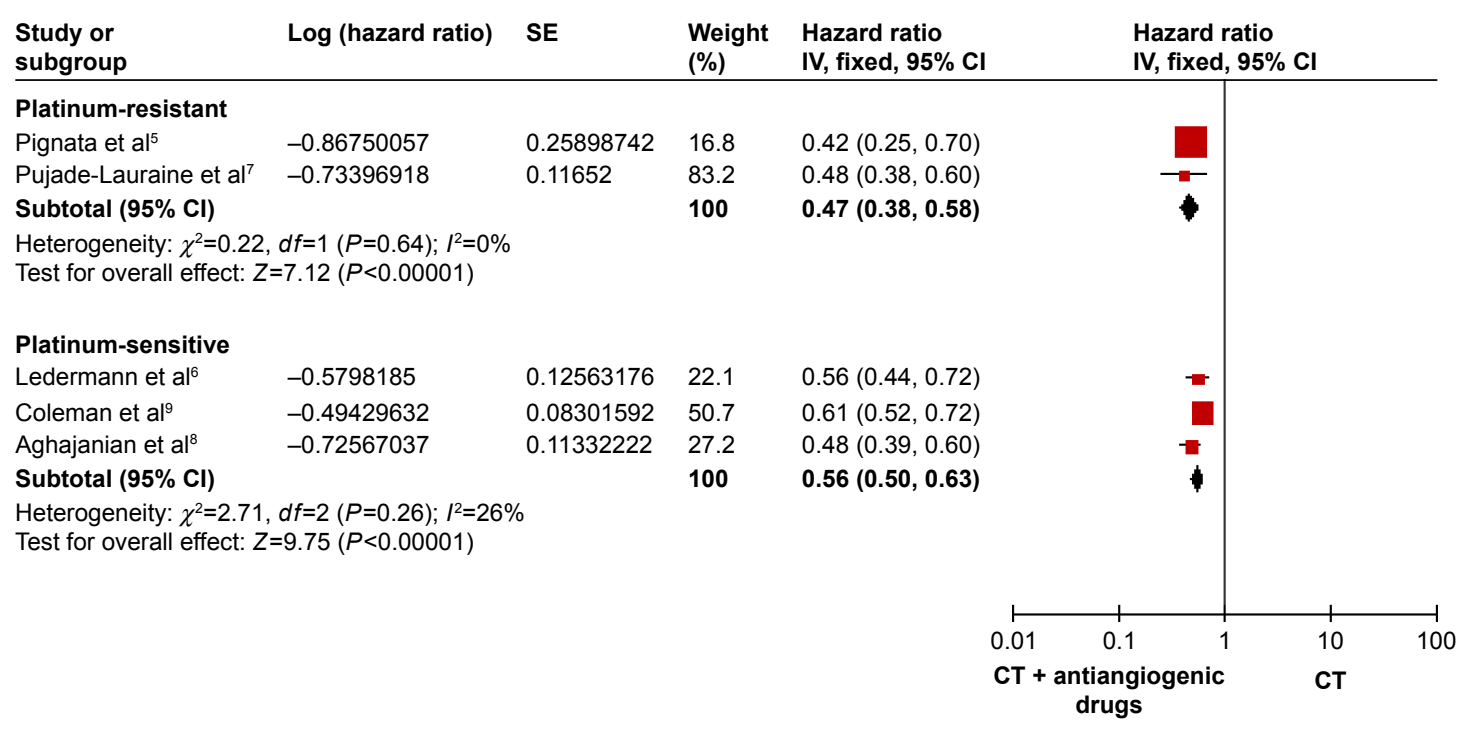

Figure 8 Forest plot for progression-free survival based on whether patients were platinum-resistant or -sensitive.

Abbreviations: SE, standard error; $\mathrm{Cl}$, confidence interval; IV, instrumental variable; CT, chemotherapy.

metastasis, and various antiangiogenic drugs have subsequently emerged. ${ }^{17-19}$

To the best of our knowledge, VEGF plays an important role in neovascularization. VEGF signals through VEGFRs and upregulates downstream signaling pathways. Another pathway uses angiopoietin, which is a regulator of tumor angiogenesis. Based on the mechanism of vascularization, we divided the RCTs into 3 groups to perform our analysis. ${ }^{10,12-14}$ In this meta-analysis, the PFS showed significant improvement with antiangiogenic therapy in those patients with relapsed ovarian cancer. The OS was clearly better in the VEGFR inhibitor, the bevacizumab and trebananib groups. In addition, both the platinum-resistant and the platinum-sensitive subgroups had a benefit in the PFS. The OS was obviously improved in the platinum-sensitive group; however, it was not statistically significant in the platinum-resistant group.

In our study, the incidences of grade $3 / 4$ toxicity were higher when compared with chemotherapy alone but were manageable; however, the type and incidence of side effects ( $\geq$ grade 3 ) varied with the different antiangiogenic drugs. Furthermore, the incidence of GIP in

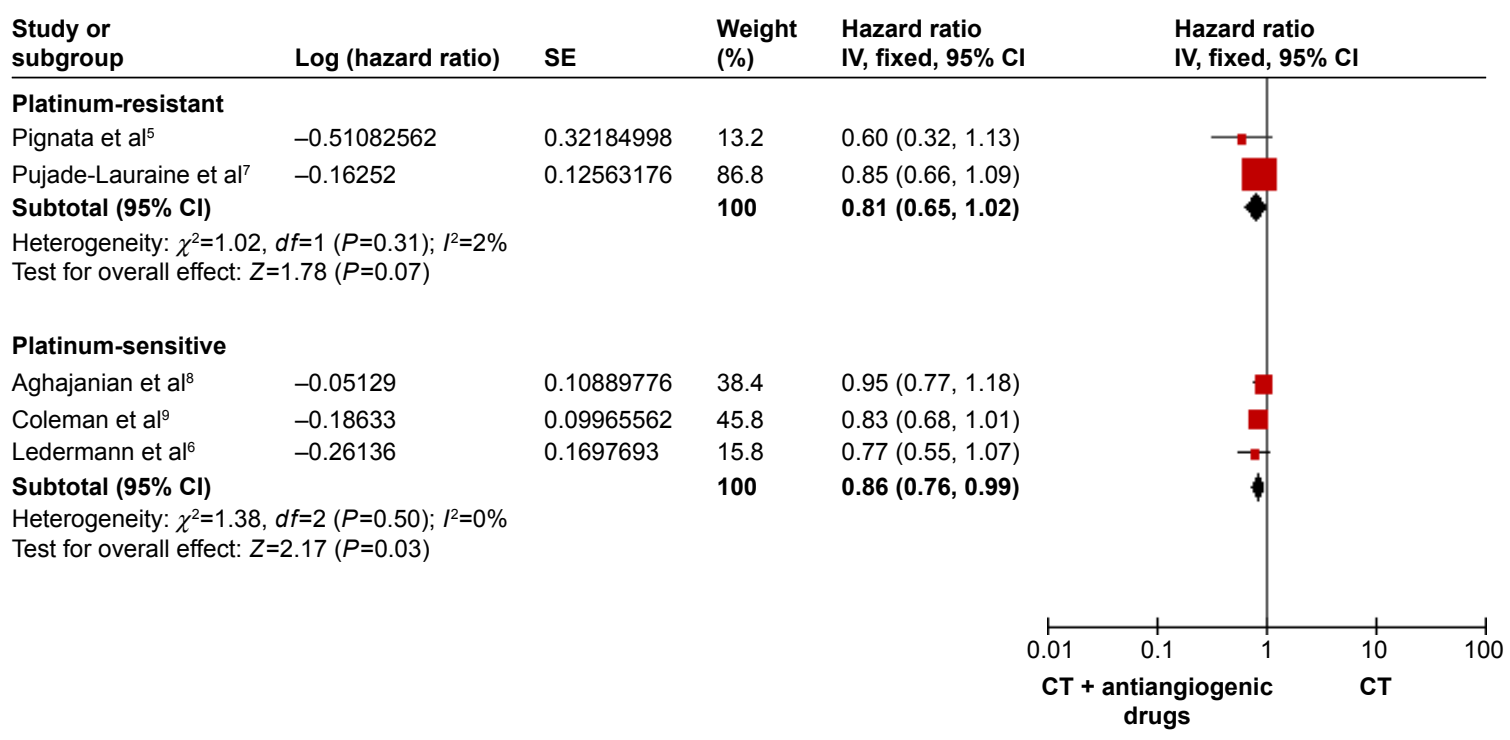

Figure 9 Forest plot for overall survival based on whether patients were platinum-resistant or -sensitive. Abbreviations: $\mathrm{SE}$, standard error; $\mathrm{Cl}$, confidence interval; $\mathrm{CT}$, chemotherapy. 
the bevacizumab group was higher than the VEGFRIs and trebananib groups. Oncologists gradually realized the significant risk of GIP. They reported that the rate of GIP in recurrent ovarian cancer varies from $0 \%$ to $11.4 \%$. However, in large Phase III clinical trials, the rate of perforation is $0 \%-2.6 \%$. GIPs carry a high rate of mortality, especially in the setting of recurrent, platinum-resistant ovarian cancer. ${ }^{20}$ Overall, the application of antiangiogenic drugs remains controversial. Bevacizumab is the most successful antiangiogenic drug, but it is not currently the upfront chemotherapy, because it has shown no statistical significance in improving the OS in recurrent ovarian cancer according to original studies..$^{3,7-9}$

Antiangiogenic therapy could be applied for recurring ovarian cancer, if the patient has previously received antiangiogenic therapy; however, the impact on the efficacy is not known. Both Huijbers's and Bergers's studies have shown the role of tumor stromal cells in the resistance to antiangiogenic drugs, and the mechanisms for use are diverse, including endothelial (progenitor) cells, smooth muscle cells, pericytes, fibroblasts, immune cells, and platelets. Multiple mechanisms develop evasive or intrinsic resistance and finally become resistant to antiangiogenic therapy. Although the incidence of resistance to antiangiogenic therapy after previous antiangiogenic therapy is not clear, it is closely related to the prognosis of the patient with recurrent ovarian cancer. Currently, there are no relevant trials in the literature. .,21,22 $^{2}$

Which recurrent patients benefit most from antiangiogenic therapy remains unclear. Abu-Jawdeh et al discovered that VEGF and its receptors were not expressed in benign ovarian tumors, with intermediate levels of expression shown in borderline ovarian tumors and strong expression shown in malignant cancer, via detection in the serum, ovarian tumor tissue, and ascites. Recently, Pasquet et al reported that ascites-derived stromal cells play a role in promoting tumor growth by inducing angiogenesis. Antiangiogenic therapy alone delays tumor tissue growth, narrows metastatic lesions, and reduces malignant peritoneal effusion. The research by Ferriss et al showed that patients with ascites treated with bevacizumab had significantly better PFS and OS, but the issue has not reached consistency. ${ }^{23-26}$

In addition to bevacizumab, there were several antiangiogenic drugs described in our meta-analysis. Therefore, the decision about selecting an appropriate antiangiogenic drug to obtain the most benefit for recurrent patients will be complicated. Both the characteristics of the patients and the toxicity of the drugs must be taken into account. Currently, only bevacizumab and pazopanib have been recommended for application in ovarian cancer by the National Comprehensive Cancer Network. ${ }^{3}$

In addition, the optimal doses and therapeutic time period have not yet been determined. In our meta-analysis, the doses and durations differed among the 8 RCTs we included in this study. The survival outcomes were improved with the use of antiangiogenic therapy as a maintenance therapy for patients with recurrent ovarian cancer. A previous meta-analysis, conducted by Qian et al, also confirmed that both the PFS and OS were improved in the antiangiogenic maintenance therapy group, when compared with the chemotherapeutic group alone. ${ }^{27,28}$ According to preclinical researches, tumor vessels were known to be leaky and dysfunctional, which disturb delivery of chemotherapy to tumor. Vascular normalization by crossover application of antiangiogenic therapy was proposed to increase the density of chemotherapeutic agents in tumor. Currently, clinical studies were not enough to prove the vascular normalization theory. Therefore, the effect of difference was still unknown between crossover and subsequent antiangiogenic therapy. ${ }^{29,30}$ In a word, a standard antiangiogenic regime has not yet been established.

Directly predicting the clinical efficacy of antiangiogenic drugs using biomarkers is currently under study, but the results do not look good. The measurement of serum-free VEGF was proposed first based on its easy accessibility and reasonable price. However, there is little predictive value as a serum biomarker due to its dynamic change between the total and free VEGF levels. Recently, 2 studies have recognized several biomarkers (mesothelin, FLT4, AGP, CA-125, and miR-378) that seem predictive of the benefits from the antiangiogenic treatment of ovarian cancer, but further larger population studies are required. ${ }^{31-33}$

Despite the fact that we only included RCTs to minimize bias in our meta-analysis, several potential limitations remain. Due to the disparate design of the research studies, characteristics of the recurrent patients, various ovarian tumor types, unclear International Federation of Gynecology and Obstetrics staging, and different doses and durations of the drugs, there was heterogeneity among all 8 studies.

\section{Conclusion}

The antiangiogenic therapy showed a clear improvement in the PFS in the treatment of relapsed ovarian cancer patients. In addition, the bevacizumab and trebananib groups showed prolonged OS. Antiangiogenesis as a targeted therapy seems to be promising, despite the many uncertainties put forth in our study. 


\section{Disclosure}

The authors report no conflicts of interest in this work.

\section{References}

1. Luvero D, Milani A, Ledermann JA. Treatment options in recurrent ovarian cancer: latest evidence and clinical potential. Ther Adv Med Oncol. 2014;6(5):229-239.

2. American Cancer Society. Cancer facts and figures 2008. Available from: http://www.cancer.org/research/cancerfactsstatistics/cancerfactsfigures2008/index. Accessed December 31, 2008.

3. National Comprehensive Cancer Network. NCCN Clinical practice Guidelines in Oncology: Ovarian Cancer Including Fallopian Tube Cancer and Primary Peritoneal Cancer. Version 2. 2015. Available from: http://www.nccn.org. Accessed 2, 2015.

4. Ledermann JA, Hackshaw A, Kaye S, et al. Randomized phase II placebo-controlled trial of maintenance therapy using the oral triple angiokinase inhibitor BIBF 1120 after chemotherapy for relapsed ovarian cancer. J Clin Oncol. 2011;29(28):3798-3804.

5. Pignata S, Lorusso D, Scambia G, et al. Pazopanib plus weekly paclitaxel versus weekly paclitaxel alone for platinum-resistant or platinum-refractory advanced ovarian cancer (MITO 11): a randomized, open-label, phase 2 trial. Lancet Oncol. 2015;16(5):561-568.

6. Ledermann JA, Embleton AC, Raja F, et al. Cediranib in patients with relapsed platinum-sensitive ovarian cancer (ICON6): a randomized, double-blind, placebo-controlled phase 3 trial. Lancet. 2016;387(10023): 1066-1074.

7. Pujade-Lauraine E, Hilpert F, Weber B, et al. Bevacizumab combined with chemotherapy for platinum-resistant recurrent ovarian cancer: the AURELIA open-label randomized phase III trial. J Clin Oncol. 2014; 32(13):1302-1308.

8. Aghajanian C, Goff B, Nycum LR, Wang YV, Husain A, Blank SV. Final overall survival and safety analysis of OCEANS, a phase 3 trial of chemotherapy with or without bevacizumab in patients with platinumsensitive recurrent ovarian cancer. Gynecol Oncol. 2015;139(1): $10-16$.

9. Coleman RL, Brady MF, Herzog TJ, et al. Gynecologic oncology. Presented at: Society of Gynecologic Oncology 2015 Annual Meeting on Women's Cancer; March 28-31, 2015; Chicago, IL, USA. Abstract 3.

10. Karlan BY, Amit MO, Gary ER, et al. Randomized, double-blind, placebo-controlled phase II study of AMG 386 combined with weekly paclitaxel in patients with recurrent ovarian cancer. J Clin Oncol. 2012; 30(4):362-371.

11. Monk BJ, Andrés P, Ignace V, et al. Anti-angiopoietin therapy with trebananib for recurrent ovarian cancer (TRINOVA-1): a randomized, multicenter, double-blind, placebo-controlled phase 3 trial. Lancet Oncol. 2014;15(8):799-808.

12. Papadopoulos N, Martin J, Ruan Q, et al. Binding and neutralization of vascular endothelial growth factor (VEGF) and related ligands by VEGF trap, ranibizumab, and bevacizumab. Angiogenesis. 2012;15(2): 171-185.

13. Holash J, Maisonpierre PC, Compton D, et al. Vessel cooption, regression, and growth in tumors mediated by angiopoietins and VEGF. Science. 1999;284(5422):1994-1998.

OncoTargets and Therapy

\section{Publish your work in this journal}

OncoTargets and Therapy is an international, peer-reviewed, open access journal focusing on the pathological basis of all cancers, potential targets for therapy and treatment protocols employed to improve the management of cancer patients. The journal also focuses on the impact of management programs and new therapeutic agents and protocols on

Submit your manuscript here: http://www.dovepress.com/oncotargets-and-therapy-journal
14. Lokadasan R, James FV, Narayanan G, Prabhakaran PK. Targeted agents in epithelial ovarian cancer: review on emerging therapies and future developments. Ecancermedicalscience. 2016;10:626.

15. Nilsson M, Heymach JV. Vascular endothelial growth factor (VEGF) pathway. $J$ Thorac Oncol. 2006;1:768-770.

16. Song F, Eustwood AJ, Gilbody S, Duley L, Sutton AJ. Publication and related biases. Health Technol Assess. 2000;4(10):1-115.

17. Coleman RL, Monk BJ, Sood AK, Herzog TJ. Latest research and treatment of advanced-stage epithelial ovarian cancer. Nat Rev Clin Oncol. 2013;10(4):211-224.

18. Cancer Genome Atlas Research Network. Integrated genomic analyses of ovarian carcinoma. Nature. 2011;474(7353):609-615.

19. Hansen JM, Coleman RL, Sood AK. Targeting the tumor microenvironment in ovarian cancer. Eur J Cancer. 2016;56:131-143.

20. McClung EC, Wenham RM. Profile of bevacizumab in the treatment of platinum-resistant ovarian cancer: current perspectives. Int $J$ Womens Health. 2016;8:59-75.

21. Huijbers EJ, van Beijnum JR, Thijssen VL, Sabrkhany S, NowakSliwinska P, Griffioen AW. Role of the tumor stroma in resistance to anti-angiogenic therapy. Drug Resist Updat. 2016;25:26-37.

22. Bergers G, Hanahan D. Modes of resistance to anti-angiogenic therapy. Nat Rev Cancer. 2008;8(8):592-603.

23. Abu-Jawdeh GM, Faix JD, Niloff J, et al. Strong expression of vascular permeability factor (vascular endothelial growth factor) and its receptors in ovarian borderline and malignant neoplasms. Lab Invest. 1996;74(6):1105-1115.

24. Yamamoto S, Konishi I, Mandai M, et al. Expression of vascular endothelial growth factor (VEGF) in epithelial ovarian neoplasms: correlation with clinicopathology and patient survival, and analysis of serum VEGF levels. Br J Cancer. 1997;76(9):1221-1227.

25. Pasquet M, Golzio M, Mery E, et al. Hospicells (ascites-derived stromal cells) promote tumorigenicity and angiogenesis. Int J Cancer. 2010;126(9):2090-2101.

26. Ferriss JS, Java JJ, Bookman MA, et al. Ascites predicts treatment benefit of bevacizumab in front-line therapy of advanced epithelial ovarian, fallopian tube, and peritoneal cancers: an NRG oncology/GOG study. Gynecol Oncol. 2015;139(1):17-22.

27. Khalique S, Hook JM, Ledermann JA. Maintenance therapy in ovarian cancer. Curr Opin Oncol. 2014;26(5):521-528.

28. Qian X, Qin J, Pan S, Li X, Pan Y, Ma S. Maintenance therapy in ovarian cancer with targeted agents improves PFS and OS: a systematic review and meta-analysis. PLoS One. 2015;10(9):e0139026.

29. Jain RK. Normalization of tumor vasculature: an emerging concept in antiangiogenic therapy. Science. 2005;307:58-62.

30. Jain RK. Normalizing tumor vasculature with anti-angiogenic therapy: a new paradigm for combination therapy. Nat Med. 2001;7:987-989.

31. Jubb AM, Harris AL. Biomarkers to predict the clinical efficacy of bevacizumab in cancer. Lancet Oncol. 2010;11(12):1172-1183.

32. Chan JK, Kiet TK, Blansit K, et al. MiR-378 as a biomarker for response to anti-angiogenic treatment in ovarian cancer. Gynecol Oncol. 2014;133(3):568-574.

33. Collinson F, Hutchinson M, Craven RA, et al. Predicting response to bevacizumab in ovarian cancer: a panel of potential biomarkers informing treatment selection. Clin Cancer Res. 2013;19(18):5227-5239.

patient perspectives such as quality of life, adherence and satisfaction. The manuscript management system is completely online and includes a very quick and fair peer-review system, which is all easy to use. Visit http://www.dovepress.com/testimonials.php to read real quotes from published authors. 